\title{
HearScreen USA
}

National Cancer Institute

\section{Source}

National Cancer Institute. hearScreen USA. NCI Thesaurus. Code C160861.

A clinically validated, US national hearing screening application (app) designed for accurate detection of hearing impairment. The screening analyzes the person's ability to perceive speech-in-noise. Based on the person's response, the app automatically generates a hearing score that indicates whether there may be a possible hearing loss. 